\title{
Upregulated EMMPRIN/CDI47 might contribute to growth and angiogenesis of gastric carcinoma: a good marker for local invasion and prognosis
}

\author{
H-C Zheng', H Takahashi', Y Murai', Z-G Cui ',2, K Nomoto', S Miwa ${ }^{3}, K_{\text {Tsuneyamaa }}^{1,2}$ and Y Takano*,I \\ 'Department of Diagnostic Pathology, Graduate School of Medical and Pharmaceutical Sciences, University of Toyama, Toyama, Japan; ${ }^{2} 2$ I st Century \\ COE Program, Graduate School of Medical and Pharmaceutical Sciences, University of Toyama, Toyama, Japan; ${ }^{3}$ Department of Internal Medicine (3), \\ Graduate School of Medical and Pharmaceutical Sciences, University of Toyama, Toyama, Japan
}

\begin{abstract}
Tumour growth depends on angiogenesis, which is closely associated with vascular endothelial growth factor (VEGF) and matrix metalloproteinases (MMPs). Extracellular MMP inducer (EMMPRIN) was reported to involve in the progression of malignancies by regulating expression of VEGF and MMPs in stromal cells. To clarify the role of EMMPRIN in progression and angiogenesis of gastric carcinoma, expression of EMMPRIN, ki-67, MMP-2, MMP-9 and VEGF was examined on tissue microarray containing gastric carcinomas $(n=234)$ and non-cancerous mucosa adjacent to carcinoma $(n=85)$ by immunohistochemistry. Additionally, microvessel density (MVD) was assessed after labelling with anti-CD34 antibody. Extracellular MMP inducer expression was compared with clinicopathological parameters of tumours, including levels of ki-67, MMP-2, MMP-9 and vascular endothelial growth factor (VEGF), MVD as well as survival time of carcinoma patients. Gastric carcinoma cell lines (HGC-27, MKN28 and MKN45) were studied for EMMPRIN expression by immunohistochemistry and Western blot. Extracellular MMP inducer expression was gradually increased from normal mucosa to carcinomas through hyperplastic or metaplastic mucosa of the stomach $(P<0.05)$. There was strong EMMPRIN expression in all gastric carcinoma cell lines despite different levels of glycosylation. Extracellular MMP inducer expression was positively correlated with tumour size, depth of invasion, lymphatic invasion, expression of ki-67, MMP-2, MMP-9 and VEGF of tumours $(P<0.05)$, but not with lymph node metastasis, UICC staging or differentiation $(P>0.05)$. Interestingly, there was a significantly positive relationship between EMMPRIN expression and MVD in gastric carcinomas $(P<0.05)$. Survival analysis indicated EMMPRIN expression to be negatively linked to favourable prognosis $(P<0.05)$, but not be independent factor for prognosis $(P>0.05)$. Further analysis showed three independent prognostic factors, depth of invasion, lymphatic and venous invasion, to influence the relationship between EMMPRIN expression and prognosis. Upregulated expression of EMMPRIN possibly contributes to genesis, growth and local invasion of gastric carcinomas. Altered EMMPRIN expression might enhance growth, invasion and angiogenesis of gastric carcinoma via upregulating MMP expression of both stromal fibroblasts and gastric cancer cells and could be considered as an objective and effective marker to predict invasion and prognosis.

British Journal of Cancer (2006) 95, I37 I-1378. doi: I0.1038/sj.bjc.6603425 www.bjcancer.com

Published online 31 October 2006

(c) 2006 Cancer Research UK
\end{abstract}

Keywords: gastric carcinoma; progression; angiogenesis; prognosis; EMMPRIN

Growth of solid tumours depends on angiogenesis, a complex and multistep process which facilitates metastasis formation by increasing the likelihood of tumour cells to enter the blood circulation, provides nutrients and oxygen for growth at the metastasis site (Rundhaug, 2005). Critical steps during tumour angiogenesis are the outgrowth of endothelial cells from preexisting capillary vessel and their migration from parental vessels under the stimulation of vascular endothelial growth factor (VEGF). Proliferating endothelial cells subsequently remodel the extracellular matrix (ECM) via matrix metalloproteinases (MMPs), align into tube-like structures, and eventually form new functional

*Correspondence: Professor Y Takano;

E-mail: ytakano@ms.toyama-mpu.ac.jp

Received I5 May 2006; revised 17 August 2006; accepted I 5 September 2006; published online 31 October 2006 blood vessels. In cancer cells, overexpression of angiogenetic factors (eg, VEGF, MMPs, etc.) results in their more secretion into the ECM to stimulate the proliferation and mobility of vascular epithelial cells, closely linked to invasion and metastasis of malignancies (Rundhaug, 2005; Ueda et al, 2005).

The search for MMP-inducing factors in tumour cells leads to the identification of extracellular MMP inducer (EMMPRIN), whose name reflects its EMMPRIN activity. Structurally, EMMPRIN is a highly glycosylated (HG) cell surface transmembrane protein which belongs to the immunoglobulin superfamily, and is composed of two immunoglobulin domains in the extracellular region, a single transmembrane domain and a short cytoplasmic domain containing 39 amino acids (Muramatsu and Miyauchi, 2003; Gabison et al, 2005a, b). Cotransfection of EMMPRIN expression vectors with different tags and crosslinking experiments have suggested that the molecules could associate with each 
other on the plasma membrane, forming homo-oligomers in a cisdependent manner via the $\mathrm{N}$-terminally located Ig-like domain (Yoshida et al, 2000; Sun and Hemler, 2001). Although EMMPRIN has a broader tissue distribution and is expressed on activated $\mathrm{T}$ cells, on differentiated macrophage, on retinal pigment epithelium, in the endometrium and in normal human keratinocytes, its expression is often elevated on breast cancers, hepatomas, oesophageal and cervical squamous cell carcinomas, colorectal and ovarian carcinomas, which increases tumour invasion by inducing MMP synthesis of surrounding stromal cells, including membrane type 1 and type $2 \mathrm{MMP}$, and the endogenous activators of MMP-2 (Davidson et al, 2003; Ishibashi et al, 2004; Marieb et al, 2004; Gabison et al, 2005a, b; Li et al, 2005; Tang et al, 2005; Zhou et al, 2005; Sier et al, 2006; van der Jagt et al, 2006). Recently, various in vivo evidences indicated that EMMPRIN might stimulate tumour angiogenesis by elevating VEGF and MMP expression in the neighbouring fibroblasts and epithelial cells in a paracrine manner (Tang et al, 2005). Taken together, it was believed that EMMPRIN had strong impact on the invasion and metastasis of malignant tumours.

Gastric carcinoma ranks the world's second leading cause of cancer mortality behind lung cancer despite a sharp worldwide decline in both its incidence and mortality since the second half of the 20th century (Kelley and Duggan, 2003). Tumorigenesis and progression of gastric carcinoma is a multistage process with the involvement of a multifactorial aetiology, which mainly results from gene-environment interactions. In our study, EMMPRIN expression was for the first time examined in gastric carcinoma and non-cancerous mucosa adjacent to carcinoma (NCMAC), and compared with its clinicopathological parameters of tumours, including expression of ki-67, MMP-2, MMP-9 and VEGF proteins as well as prognosis to explore the roles of EMMPRIN in stepwise development of gastric carcinoma.

\section{MATERIALS AND METHODS}

\section{Subjects}

Gastric carcinomas $(n=234)$ and NCMACs $(n=85,38$ cases of normal mucosa and 47 cases of hyperplastic or metaplastic mucosa) were collected from our affiliated hospital and related institutes between 1993 and 2002. The patients with gastric carcinoma were 170 men and 64 women $(38-88$ years, mean $=66.8$ years). Among them, 104 cases have tumours accompanied with lymph node metastasis. None of the patients underwent chemotherapy or radiotherapy before surgery. They all provided consent for use of tumour tissue for clinical research and our University Ethical Committee approved the research protocol. We followed up carcinoma patients by consulting their case documents and telephoning.

\section{Pathology}

All tissues were fixed in $4 \%$ neutralised formaldehyde, embedded in paraffin and incised into $4 \mu \mathrm{m}$ sections. These sections were stained by haematoxylin and eosin (HE) to confirm their histological diagnosis and other microscopic characteristics. The staging for each gastric carcinoma was evaluated according to the Union Internationale Contre le Cancer (UICC) system for the extent of tumour spread (Sobin and Wittekind, 2002). Furthermore, tumour size, depth of invasion, lymphatic and venous invasion, and differentiation were determined.

\section{Tissue microarray}

Representative areas of solid tumours were identified in HEstained sections of the selected tumour cases and a $2 \mathrm{~mm}$ in-diameter tissue core per donor block was punched out and

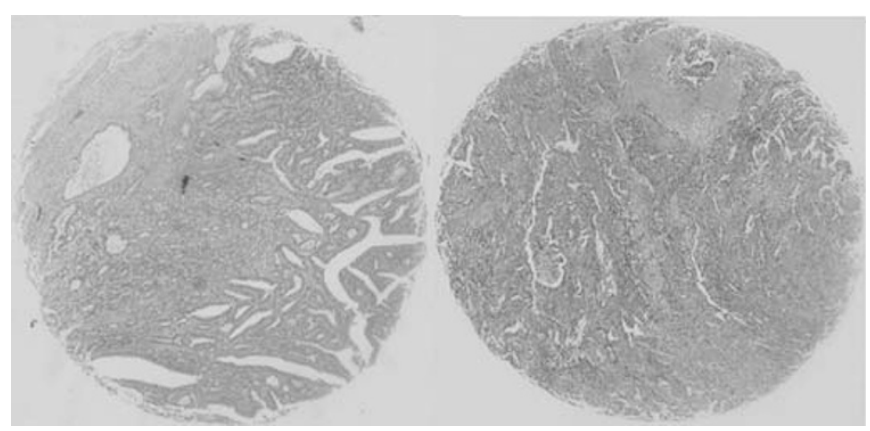

Figure I Haematoxylin and eosin staining on the tissue microarray of gastric carcinoma.

transferred to a recipient block with a maximum of 48 cores using a Tissue Microarrayer (AZUMAYA KIN-1, Japan). Four- $\mu$ m-thick sections were consecutively incised from the recipient block and transferred to poly-lysine-coated glass slides. Haematoxylin and eosin staining was performed on tissue microarray for confirmation of tumour tissue (Figure 1).

\section{Cell lines and culture}

Gastric carcinoma cell lines, HGC-27 (undifferentiated adenocarcinoma), MKN28 (well-differentiated adenocarcinoma) and MNK45 (poorly differentiated adenocarcinoma), come from Japanese Physical and Chemical Institute. They were maintained in RPMI 1640 (MKN28 and MKN45) or MEM (HGC-27) medium supplemented with $10 \%$ foetal bovine serum, $100 \mathrm{U} \mathrm{ml}^{-1}$ penicillin and $100 \mu \mathrm{g} \mathrm{ml}^{-1}$ streptomycin, in a humidified atmosphere of $5 \%$ $\mathrm{CO}_{2}$ at $37^{\circ} \mathrm{C}$. Total protein was prepared from all cells by cell disruption buffer according to PARIS manual (Ambion Inc, Austin, Texas, USA). All cells were collected by centrifugation, rinsed with phosphate-buffered saline, fixed by $10 \%$ formalin and then embedded in paraffin.

\section{Immunohistochemistry}

Consecutive sections were deparaffinised with xylene, dehydrated with alcohol and subjected to antigen retrieval by irradiating in target retrieval solution (TRS, Dako, Carpinteria, CA 93013, USA) for $5 \mathrm{~min}$ with microwave oven (Oriental rotor Lmt. Co. Japan). Five per cent bovine serum albumin was then applied for $1 \mathrm{~min}$ to prevent nonspecific binding. The sections were incubated with primary antibodies for $15 \mathrm{~min}$, then treated with the anti-mouse or anti-rabbit Envison-PO (Dako, USA) antibodies for $15 \mathrm{~min}$. All the incubations were performed in a microwave oven to allow intermittent irradiation as described previously (Kumada et al, 2004). After each treatment, the slides were washed with TBST (10 mM Tris- $\mathrm{HCl}, 150 \mathrm{~mm} \mathrm{NaCl}, 0.1 \%$ Tween 20 ) three times for $1 \mathrm{~min}$. Mouse anti-EMMPRIN (Novocastro, New Castle upon Tyne NE 1282W, UK; 1:50), rabbit anti-ki-67 (Dako, USA; $1: 25$ ), mouse anti-MMP-2 (Daiichi Fine Chemical. Co. Lt, Japan; 1:50), mouse anti-MMP-9 (Daiichi Fine Chemical. Co. Lt, Japan; $1: 150$ ), rabbit anti-VEGF (LAB VISION, Fremont CA 94530, USA; ready to use), and mouse anti-CD34 (Dako, USA; 1:100) antibodies were employed for the detection of the respective proteins. Binding sites were visualised with 3,3'-diaminobenzidine. After counterstained with Mayer's haematoxylin, the sections were dehydrated, cleared and mounted. Omission of the primary antibody was used as a negative control and appropriate positive controls were utilised as recommended by the manufacturers.

\section{Evaluation of immunohistochemistry}

The immunoreactivity to EMMPRIN was localised in the cytoplasm and membrane, ki-67 in the nucleus, and MMP-2, 

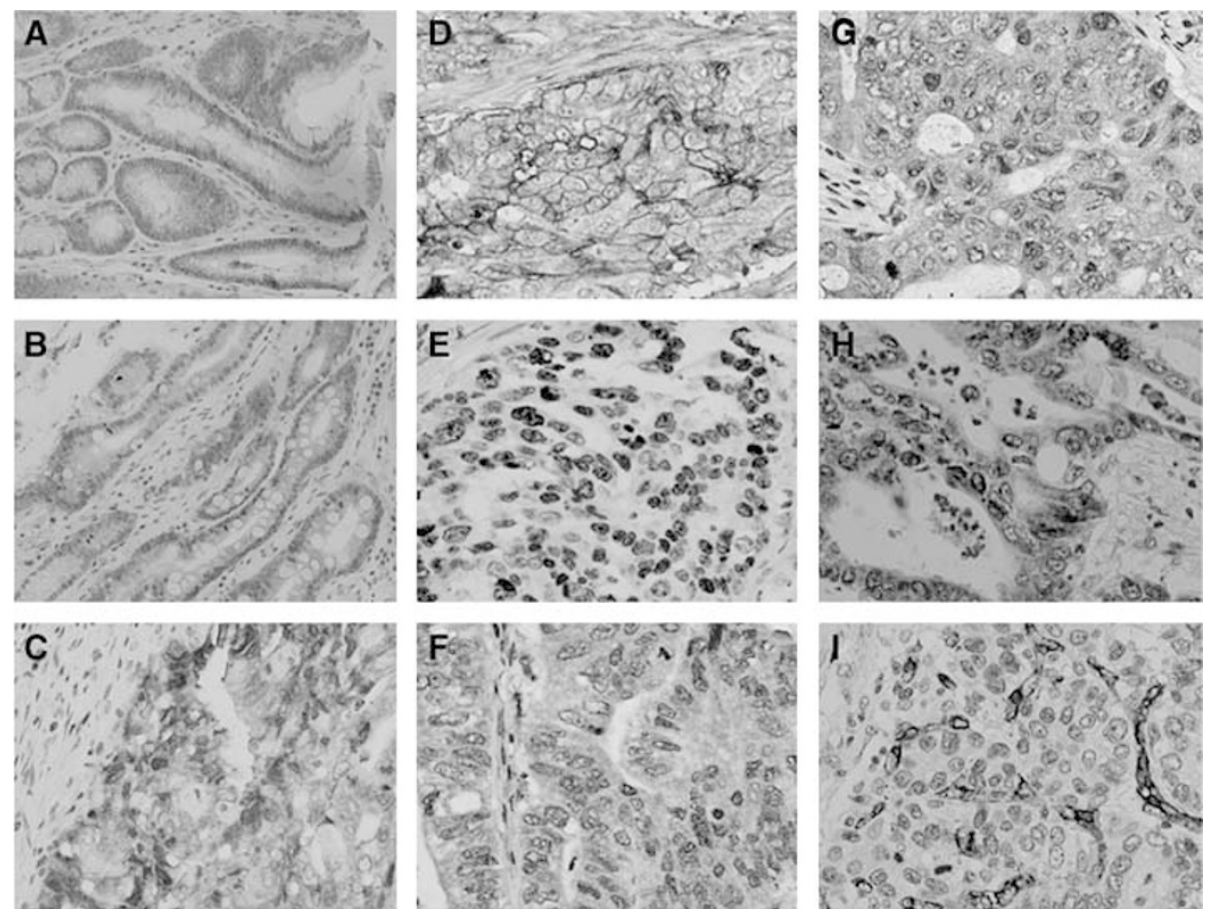

Figure 2 Immunostaining in gastric carcinoma and non-cancerous mucosa. Note EMMPRIN expression limited to cytoplasm and membrane in hyperplastic $(\mathbf{A})$ and metaplastic (B) NCMAC and carcinoma (C, D) of the stomach. Ki-67 (E) distributed to the nucleus of gastric carcinoma cells, MMP-2 $(\mathbf{F}), \operatorname{MMP}-9(\mathbf{G})$ and VEGF $(\mathbf{H})$ to the cytoplasm, and CD34 (I) in the membrane and cytoplasm of vascular epithelial cell to label MVD.

MMP-9 and VEGF only in the cytoplasm (Figure 2). One hundred cells were randomly selected and counted from five representative fields of each section blindly by three independent observers (Takano Y, Tsuneyama K and Zheng HC). The positive percentage of counted cells was graded semiquantitatively according to a fourtier scoring system: negative $(-), 0 \sim 5 \%$; weakly positive $(+)$, $6 \sim 25 \%$; moderately positive $(++), 26 \sim 50 \%$ and strongly positive $(+++), 51 \sim 100 \%$.

\section{Microvessel density counting}

CD34 expression in the cytoplasm and membrane of vascular epithelial cells was selected for the microvessel density (MVD) counting, although it was occasionally localised in tumour cells and fibroblasts. A modified Weidner's method was used to calculate the MVD of gastric carcinoma after anti-CD34 immunostaining (Weidner, 1995). In brief, observers selected five representative areas and counted individual microvessels on a $\times 400$ field $\left(0.1885 \mathrm{~mm}^{2}\right.$ per field) after the area of highest neovascularisation was identified, Any brown staining endothelial cell or endothelial cell cluster that was clearly separated from the adjacent microvessel, tumour cells and other connective tissue elements was considered as a single, countable microvessel. The counts were performed independently by two investigators.

\section{Western blot}

Fifty micrograms of denatured protein was separated on an SDSpolycrylamide gel (10\% acrylamide) and transferred to Hybond membrane (Amersham, Germany), which was then blocked overnight in 5\% milk in TBST. For immunobloting, the membrane was incubated for $1 \mathrm{~h}$ with the antibody against EMMPRIN (Novocastra, UK, 1:100). Then, it was rinsed by TTBS and incubated with anti-mouse IgG conjugated to horseradish peroxidase (Dako, USA, $1: 1000$ ) for $1 \mathrm{~h}$. Bands were visualised with X-ray film (Fujifilm, Japan) by Amersham ECL-Plus detection reagents (Amersham, Germany). After that, membrane was washed with WB Stripping Solution ( $\mathrm{pH} 2-3$, Nacalai, Japan) for $1 \mathrm{~h}$ and treated as described above, except mouse tubulin- $\alpha$ antibody (Dako, USA, 1:100) as internal control.

\section{Statistical analysis}

Statistical evaluation was performed using Spearman correlation test to analyse the rank data and Kruskal-wallis $H$ test to differentiate non-parametric means of different groups. KaplanMeier survival plots were generated and comparisons between survival curves were made with the log-rank statistic. The Cox's proportional hazard model was employed for multivariate analysis. $P<0.05$ was considered as statistically significant. SPSS 10.0 software was employed to analyse all data.

\section{RESULTS}

\section{Extracellular MMP inducer expression in gastric carcinomas}

Extracellular MMP inducer expression was evident in the cytoplasm and membrane of hyperplastic, metaplastic epithelial cells of NCMAC and cancerous cells of stomach (Figure 2A, B). As showed in Table 1, EMMPRIN expression was increased from gastric normal mucosa to carcinoma through hyperplastic or metaplastic mucosa $(P<0.05)$. As summarised in Table 2, EMMPRIN expression showed a significantly positive association with tumour size, depth of invasion, lymphatic invasion, expression of ki-67, MMP-2, MMP-9 and VEGF of tumours $(P<0.05)$, but not with lymph node metastasis, UICC staging or differentiation $(P>0.05)$. Interestingly, there was a significantly positive relationship between EMMPRIN expression and MVD in the gastric carcinomas $(P<0.05)$ (Table 3$)$. 
Table I EMMPRIN expression in gastric NCMAC and carcinoma

EMMPRIN expression

\begin{tabular}{lcccccccc}
\cline { 3 - 7 } Groups & $\mathbf{n}$ & - & $\boldsymbol{+}$ & $\mathbf{+ +}$ & $\mathbf{+ + +}$ & PR (\%) & rs & P-value \\
\hline Normal mucosa & 38 & 26 & 10 & 2 & 0 & 31.6 & 0.288 & $<0.0001$ \\
Hyperplastic or metaplastic mucosa & 47 & 17 & 20 & 9 & 1 & 63.8 & \\
Carcinoma & 234 & 82 & 51 & 47 & 54 & $65.0 *$ & \\
\hline
\end{tabular}

EMMPRIN = extracellular matrix metalloproteinase inducer; NCMAC = non-cancerous mucosa adjacent to carcinoma; PR = positive rate; $r s=$ Spearmen correlation coefficient.

Table 2 Relationship between EMMPRIN expression and clinicopathological features of gastric carcinoma

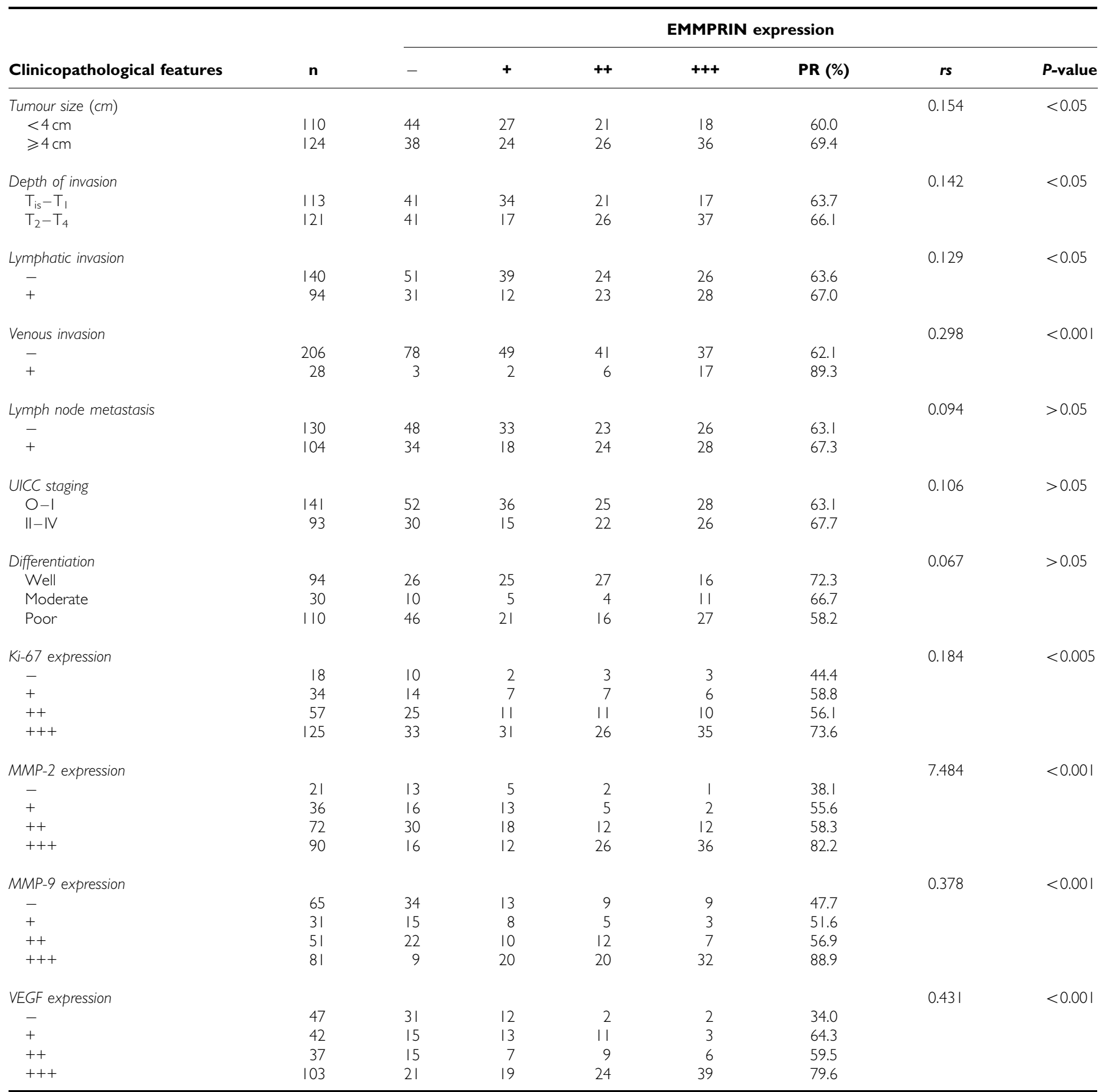

EMMPRIN = extracellular matrix metalloproteinase inducer, $\mathrm{PR}=$ positive rate; $\mathrm{MMP}=$ matrix metalloproteinase; $r$ s: Spearmen correlation coefficient; $\mathrm{T}_{\text {is }}=$ carcinoma in situ;

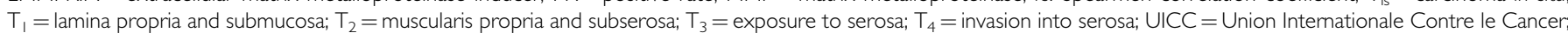
$\mathrm{VEGF}=$ vascular endothelial growth factor. 
Three gastric cell lines exhibited strong EMMPRIN expression according to immunostaining data (Figure 3). Extracellular MMP inducer exists in tumour and transformed cell lines as HG form migrating at $\sim 45-65 \mathrm{kDa}$ and as a less glycosylated (LG) form migrating at $\sim 32-44 \mathrm{kDa}$, depending on the glycosylation of the core protein $(27 \mathrm{kDa})$ (Riethdorf et al, 2006; Vigneswaran et al, 2006). Western blot also indicated these cell lines strongly expressed EMMPRIN, but the HG form in all cell lines and LG one strongly in HGC-27 and MKN45 and weakly in MKN28 (Figure 4).

Table 3 Relationship between EMMPRIN expression and MVD in gastric carcinoma

\begin{tabular}{lccc}
\hline EMMPRIN expression & $\boldsymbol{n}$ & MVD (mean \pm s.d.) & $P$-value \\
\hline- & 82 & $30.4 \pm 18.7$ & $<0.05$ \\
+ & 50 & $28.6 \pm 12.6$ & \\
++ & 47 & $36.1 \pm 17.9$ & \\
+++ & 54 & $38.9 \pm 23.0$ & \\
Total & 233 & $33.1 \pm 18.9$ & \\
\hline
\end{tabular}

EMMPRIN = extracellular matrix metalloproteinase inducer; $M V D=$ microvesse density; s.d., standard deviation.

\section{Univariate and multivariate survival analysis}

Follow-up information was available on 219 patients with gastric carcinoma for period ranging from 0.2 months to 12.2 years (mean $=40.4$ months). Figure 5 showed the survival curves stratified according to EMMPRIN expression. Univariate analyses using Kaplan-Meier method indicated that cumulative rate of the patients with negative EMMPRIN expression was significantly higher than that with its weakly, moderately and strongly positive expression $(P<0.05)$. Multivariate analysis using the Cox's proportional hazard model indicated that depth of invasion, lymphatic and venous invasion, but not EMMPRIN, were independent prognostic factors (Table 4). Further analysis showed that these three locally invasive factors to have effects on the relationship between EMMPRIN expression and survival time of the carcinoma patients (Table 5).

\section{DISCUSSION}

The microenvironment of the tumour - host interface is involved in many processes impacting on tumour development, including angiogenesis, growth, dissemination and metastasis of tumours. The crosstalk interaction between tumour cells and adjacent stromal cells participates in tumour immune escape, spreading and angiogenesis, which is conducted by a number of soluble and membrane molecules, including soluble Fas, Fas ligand, soluble
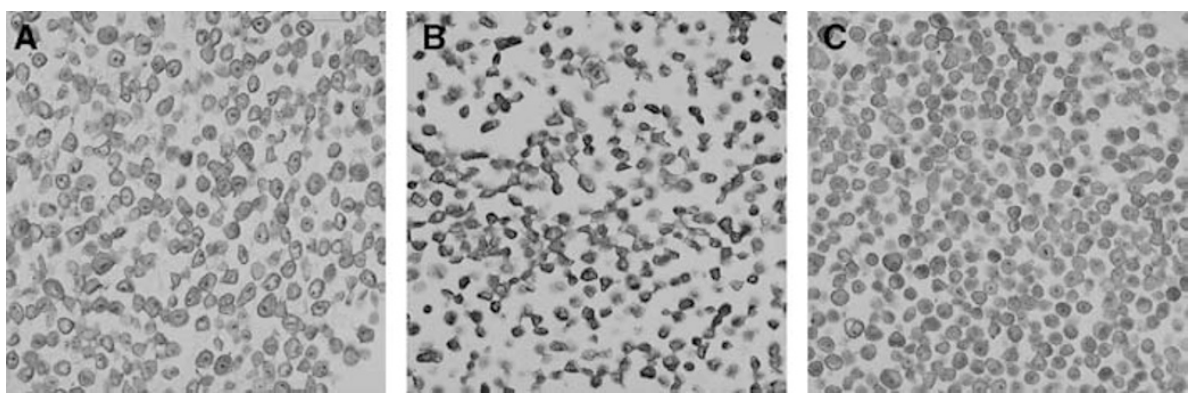

Figure 3 Extracellular MMP inducer immunostaining in gastric carcinoma cell lines. (A) HGC-27; (B) MKN28; (C) MKN45.

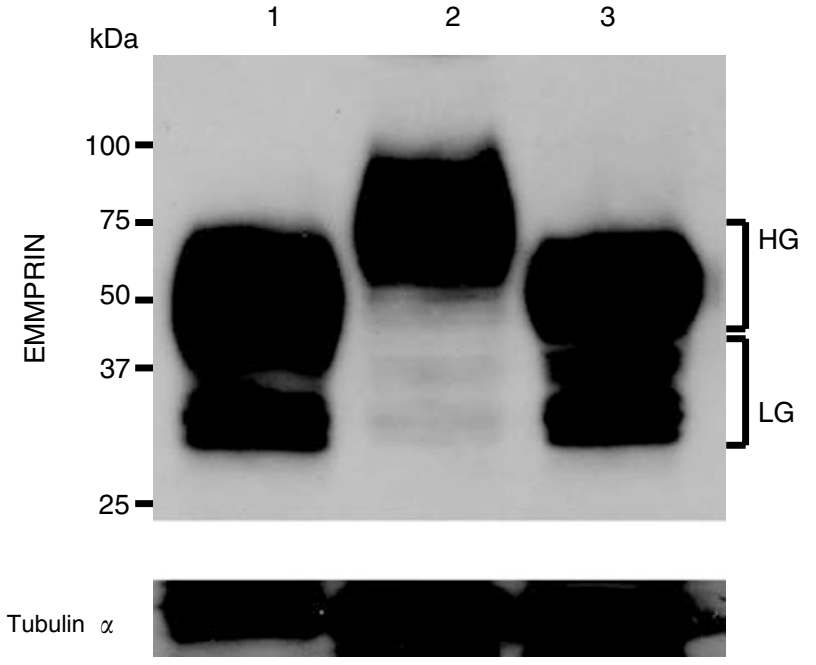

Figure 4 Western blot analysis of EMMPRIN in gastric carcinoma cell lines. Cell lysate was loaded and probed with monoclonal mouse antihuman EMMPRIN antibody with tubulin- $\alpha$ as an internal control. Lane no. I: HGC-27; no. 2 MKN28; no. 3 MKN45. HG: highly glycosylated form ( 45-65 kDa); LG: less glycosylated form ( 32-44 kDa).

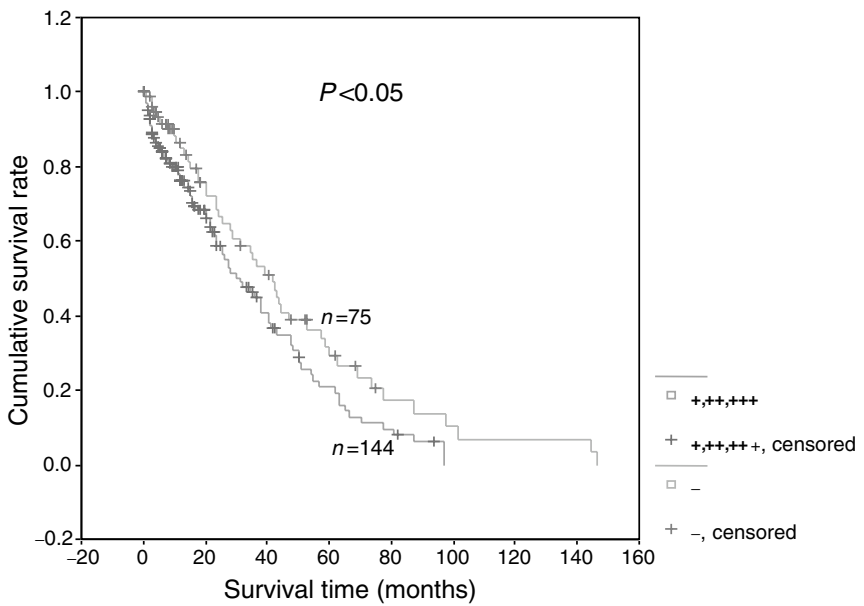

Figure 5 Correlation between status of EMMRIN and prognosis of the gastric carcinoma patients. Kaplan-Meier curves for cumulative survival rate of patients with gastric carcinomas according to EMMPRIN expression. 
Table 4 Multivariate analysis of clinical variables for gastric carcinomas

\begin{tabular}{|c|c|c|c|}
\hline Number & Clinicopathological parameters & Relative risk $(95 \% \mathrm{CI})$ & $P$-value \\
\hline A & Tumour size $(\geqslant 4 \mathrm{~cm})$ & $1.496(0.792-2.826)$ & $>0.05$ \\
\hline B & Depth of invasion $\left(T_{i s, 1} / T_{2,3}\right)$ & $3.635(1.501-8.804)$ & $<0.05$ \\
\hline C & Lymphatic invasion $(-/+)$ & $1.576(1.222-2.032)$ & $<0.05$ \\
\hline $\mathrm{D}$ & Venous invasion $(-/+)$ & $1.539(1.068-2.218)$ & $<0.05$ \\
\hline$E$ & Lymph node metastasis $(-/+)$ & $2.635(0.913-7.607)$ & $>0.05$ \\
\hline $\mathrm{F}$ & UICC staging $(\mathrm{O}-\mathrm{I} / \mathrm{II}-\mathrm{IV})$ & $0.429(0.143-1.284)$ & $>0.05$ \\
\hline G & Differentiation (Well/moderately/poorly) & $1.014(0.778-1.322)$ & $>0.05$ \\
\hline $\mathrm{H}$ & Ki-67 expression $(-/+/++/++++)$ & $0.822(0.619-1.092)$ & $>0.05$ \\
\hline । & MMP-2 expression $(-/+/++/++++)$ & $0.978(0.706-1.357)$ & $>0.05$ \\
\hline J & MMP-9 expression $(-/+/++/++++)$ & $1.028(0.836-1.264)$ & $>0.05$ \\
\hline K & VEGF expression $(-/+/++/++++)$ & $0.957(0.748-1.225)$ & $>0.05$ \\
\hline L & MVD & $1.001(0.987-1.014)$ & $>0.05$ \\
\hline M & EMMPRIN expression $(-/+\sim++++)$ & $1.055(0.848-1.313)$ & $>0.05$ \\
\hline
\end{tabular}

$\mathrm{Cl}=$ confidence interval; $\mathrm{EMMPRIN}=$ extracellular matrix metalloproteinase inducer; $\mathrm{MMP}=$ matrix metalloproteinase; $\mathrm{MVD}=$ microvessel density; $\mathrm{UICC}=\mathrm{Union}$ Internationale Contre le Cancer; VEGF = vascular endothelial growth factor.

Table 5 Multivariate analysis of EMMPRIN expression and other concordant factors in gastric carcinomas

\begin{tabular}{lcc}
\hline Groups & Relative risk $\mathbf{( 9 5 \%} \mathbf{C l})$ & $P$-value \\
\hline$M$ & $1.191(0.917-1.415)$ & $<0.05$ \\
$M+B$ & $1.085(0.917-1.285)$ & $>0.05$ \\
$M+C$ & $1.084(0.912-1.290)$ & $>0.05$ \\
$M+D$ & $1.042(0.860-1.263)$ & $>0.05$ \\
$M+B+C$ & $1.052(0.887-1.248)$ & $>0.05$ \\
$M+B+D$ & $0.990(0.820-1.195)$ & $>0.05$ \\
$M+C+D$ & $1.008(0.837-1.214)$ & $>0.05$ \\
$M+B+C+D$ & $0.982(0.816-1.181)$ & $>0.05$ \\
\hline
\end{tabular}

$\mathrm{Cl}=$ confidence interval; EMMPRIN = extracellular matrix metalloproteinase inducer.

MMPs, soluble VEGF and EMMPRIN (Zheng et al, 2003; Tang et al, 2005).

Numerous studies have indicated that the presence and modulation of EMMPRIN might play some role in the normal physiological processes (Gabison et al, 2005a, b). In the present study, it was found that EMMPRIN was positively expressed in hyperplastic or metaplastic epithelium of gastric NCMAC. Gabison et al $(2005 \mathrm{a}, \mathrm{b})$ also reported EMMPRIN to be predominantly expressed in corneal epithelium but markedly elevated in the anterior stroma of ulcerated corneas. Therefore, we speculate that EMMPRIN might be involved in stromal remodelling and epithelial repair after injury. Compared with gastric normal, hyperplastic or metaplastic mucosa, gastric carcinoma highly expressed EMMPRIN protein in line with other malignancies (Davidson et al, 2003; Ishibashi et al, 2004; Marieb et al, 2004; Li et al, 2005; Tang et al, 2005; Zhou et al, 2005; Sier et al, 2006; van der Jagt et al, 2006). Additionally, strong expression was also observed in gastric carcinoma cell lines in our study. Experimental overexpression of EMMPRIN in relatively less aggressive carcinoma cells lines results in an ability to form large and malignant tumours with a more invasive phenotype in the nude mice (Toole, 2003). These findings suggest that upregulation of EMMPRIN plays an essential role in the malignant transformation of gastric epithelial cells.

Elevated EMMPRIN expression has also been shown to correlate with the progression of various malignancies (Davidson et al, 2003; Ishibashi et al, 2004; Marieb et al, 2004; Gabison et al, 2005a, b; Li et al, 2005; Tang et al, 2005; Zhou et al, 2005; Sier et al, 2006; van der Jagt et al, 2006). Our results indicated that EMMPRIN overexpression was positively linked to tumour size of gastric carcinoma. To explore the mechanism about the regulatory effect of EMMPIN on tumour growth, we examined expression of ki-67, a good proliferation marker and consequently found a positive association between the two parameters. Yang et al (2006) found that EMMPRIN expression in breast carcinoma cells rendered them resistant to anoikis, a form of apoptosis triggered by a lack of or improper cell-matrix interactions, mediated by downregulation of the proapoptotic BH3-only protein, Bim, through an MAP kinase-dependent pathway. Marieb et al (2004) documented that upregulated EMMPRIN expression stimulates hyaluronan production by elevating hyoluronan synthases, which is closely related to the anchorage-independent growth of cancer cells. Taken together, our result supported the opinion that EMMPRIN might enhance tumour growth of gastric carcinomas by disrupting the balance between apoptosis and proliferation.

Our results showed no association between EMMPRIN expression and carcinoma differentiation, although its higher expression was found in intestinal-type gastric carcinoma (Zheng et al, 2006a, b). The discrepancies might be largely attributable to different grouping and statistical methods. For example, the Spearman correlation analysis was employed to analyse the rank data in the present study, which could differentiate not only positive rate but also expression level. It was worth noting that LG EMMPRIN was weaker in the well-differentiated cell line (MKN28) than the poorly differentiated (MNK45) or undifferentiated ones (HGC-27), although there was no difference in its expression level from our statistical results of immunohistochemistry in gastric carcinoma, indicating that EMMPRIN glycosylation might be linked to carcinoma differentiation.

In our study, it was found that gastric carcinomas with higher EMMPRIN expression displayed more ability to invade into lymphatic or venous vessels, or through the gastric wall. A large body of evidence indicates that increased soluble EMMPRIN released from cancer cells can stimulate the MMP synthesis and activation of surrounding stromal cells so as to promote the progression of tumours (Yoshida et al, 2000; Sun and Hemler, 2001; Davidson et al, 2003; Muramatsu and Miyauchi, 2003; Ishibashi et al, 2004; Marieb et al, 2004; Gabison et al, 2005a, b; Li et al, 2005; Tang et al, 2005; Zhou et al, 2005; Sier et al, 2006; van der Jagt et al, 2006). Recently, Tang et al (2004) indicated that elevation of MMPs mediated by EMMPRIN could result in more proteolytic cleavage of membrane-associated EMMPRIN, forming a positive feedback tumour-stoma interaction. Furthermore, EMMPRIN transfection of tumour cells or treating tumour cells with the recombinant protein increased the expression of MMPs, especially MMP-2 (Sun and Hemler, 2001), as also evidenced by the positive correlation of EMMPRIN expression with MMP-2 and MMP-9 expression in our cases of gastric carcinoma. Our group also found the negative association between expression of EMMPRIN and ECM tenascin, possibly owing to its regulatory effect on MMP secretion (Zheng et al, 2006a, b). It was suggested 
that upregulated EMMPRIN expression possibly contributes to local invasion by stimulating the MMP expressions of both stromal and tumour cells in gastric carcinomas.

Tumour growth depends not only on apoptotic suppression, but also angiogenesis. In the present study, we found that there was a positive relationship between EMMPRIN expression and MVD in gastric carcinoma. A recent study indicated that EMMPRIN could enhance VEGF expression of stromal fibroblasts and carcinoma cells to stimulate tumour angiogenesis via the PI3K-Akt signalling pathway (Tang et al, 2005, 2006). Our results also showed that EMMPRIN expression was positively correlated with VEGF expression in gastric carcinoma. The EMMPRIN-induced MMP expression in cancer cells also had impact on the tumour angiogenesis (Sun and Hemler, 2001). In combination of these evidences, it was suggested that EMMPRIN-mediated angiogenesis might participate in growth and spread of gastric carcinoma.

Although Ishibashi et al (2004) reported that EMMPRIN expression was not associated with the recurrence-free survival of oesophageal squamous cell carcinoma, Davidson et al (2003) found that EMMPRIN was a good prognostic marker in ovarian carcinoma. To further clarify the clinicopathological significance, we analysed the relation of EMMPRIN expression with survival of 219 patients with gastric carcinoma. The results revealed a link between loss and favourable survival, albeit not independent of other parameters. The multivariate analysis demonstrated three independent prognostic factors, depth of invasion, lymphatic and venous invasion, which affected the relationship between EMMPRIN expression and prognosis.

In conclusion, upregulated expression of EMMPRIN might contribute to tumorigenesis, growth and local invasion of gastric carcinoma. Altered EMMPRIN expression might enhance invasion and angiogenesis via upregulating MMP and VEGF expression of both stromal fibroblasts or gastric carcinoma cells. It could thus be considered as an objective and effective marker to predict the invasion and prognosis of gastric carcinoma. The regulatory effects of EMMPRIN on VEGF in gastric carcinoma should be clarified in the further study.

\section{ACKNOWLEDGEMENTS}

We particularly thank Kanako Yasuyoshi, Tokimasa Kumada and Hideki Hatta for their technical help and Yukari Inoue for her secretarial assistance. This work was partially supported by the Japanese Ministry of Education, Science, Sports and Culture, Grant-in-Aid for Scientific Research 14770072 Japanese Smoking Society.

\section{REFERENCES}

Davidson B, Givant-Horwitz V, Lazarovici P, Risberg B, Nesland JM, Trope CG, Schaefer E, Reich R (2003) Matrix metalloproteinases (MMP), EMMPRIN (extracellular matrix metalloproteinase inducer) and mitogen-activated protein kinases (MAPK): co-expression in metastatic serous ovarian carcinoma. Clin Exp Metastas 20: 621-631

Gabison EE, Hoang-Xuan T, Mauviel A, Menashi S (2005a) EMMPRIN/ CD147, an MMP modulator in cancer, development and tissue repair. Biochimie 87: $361-368$

Gabison EE, Mourah S, Steinfels E, Yan L, Hoang-Xuan T, Watsky MA, De Wever B, Calvo F, Mauviel A, Menashi S (2005b) Differential expression of extracellular matrix metalloproteinase inducer (CD147) in normal and ulcerated corneas: role in epithelio-stromal interactions and matrix metalloproteinase induction. Am J Pathol 166: 209-219

Ishibashi Y, Matsumoto T, Niwa M, Suzuki Y, Omura N, Hanyu N, Nakada K, Yanaga K, Yamada K, Ohkawa K, Kawakami M, Urashima M (2004) CD147 and matrix metalloproteinase-2 protein expression as significant prognostic factors in esophageal squamous cell carcinoma. Cancer 101: 1994-2000

Kelley JR, Duggan JM (2003) Gastric cancer epidemiology and risk factors. $J$ Clin Epidemiol 56: $1-9$

Kumada T, Tsuneyama K, Hatta H, Ishizawa S, Takano Y, Takano K (2004) Improved 1-h rapid immunostaining method using intermittent microwave irradiation: practicability based on 5 years application in Toyama Medical and Pharmaceutical University Hospital. Mod Pathol 17: $1141-1149$

Li HG, Xie DR, Shen XM, Li HH, Zeng H, Zeng YJ (2005) Clinicopathological significance of expression of paxillin, syndecan-1 and EMMPRIN in hepatocellular carcinoma. World J Gastroenterol 11: 1445-1451

Marieb EA, Zoltan-Jones A, Li R, Misra S, Ghatak S, Cao J, Zucker S, Toole BP (2004) Emmprin promotes anchorage-independent growth in human mammary carcinoma cells by stimulating hyaluronan production. Cancer Res 64: 1229-1232

Muramatsu T, Miyauchi T (2003) Basigin (CD147): a multifunctional transmembrane protein involved in reproduction, neural function, inflammation and tumor invasion. Histol Histopathol 18: $981-987$

Riethdorf S, Reimers N, Assmann V, Kornfeld JW, Terracciano L, Sauter G, Pantel K (2006) High incidence of EMMPRIN expression in human tumors. Int J Cancer 119: 1800 - 1810

Rundhaug JE (2005) Matrix metalloproteinases and angiogenesis. J Cell Mol Med. 9: 267-285

Sier CF, Zuidwijk K, Zijlmans HJ, Hanemaaijer R, Mulder-Stapel AA, Prins FA, Dreef EJ, Kenter GG, Fleuren GJ, Gorter A (2006) EMMPRIN-induced MMP-2 activation cascade in human cervical squamous cell carcinoma. Int J Cancer 118: $2991-2998$

Sobin LH, Wittekind CH (2002) TNM Classification of Malignant Tumours, 6 th edn. New Jersey: John Wiley \& Sons, Hoboken

Sun J, Hemler ME (2001) Regulation of MMP-1 and MMP-2 production through CD147/extracellular matrix metalloproteinase inducer interactions. Cancer Res 61: 2276-2281

Tang Y, Kesavan P, Nakada MT, Yan L (2004) Tumor-stroma interaction: positive feedback regulation of extracellular matrix metalloproteinase inducer (EMMPRIN) expression and matrix metalloproteinase-dependent generation of soluble EMMPRIN. Mol Cancer Res 2: 73-80

Tang Y, Nakada MT, Kesavan P, McCabe F, Millar H, Rafferty P, Bugelski P, Yan L (2005) Extracellular matrix metalloproteinase inducer stimulates tumor angiogenesis by elevating vascular endothelial cell growth factor and matrix metalloproteinases. Cancer Res 65: 3193-3199

Tang Y, Nakada MT, Rafferty P, Laraio J, McCabe FL, Millar H, Cunningham M, Snyder LA, Bugelski P, Yan L (2006) Regulation of vascular endothelial growth factor expression by EMMPRIN via the PI3K-Akt signaling pathway. Mol Cancer Res 4: 371-377

Toole BP (2003) Emmprin (CD147), a cell surface regulator of matrix metalloproteinase production and function. Curr Top Dev Biol 54: 371-389

Ueda M, Terai Y, Kanda K, Kanemura M, Takehara M, Futakuchi H, Yamaguchi H, Yasuda M, Nishiyama K, Ueki M (2005) Tumor angiogenesis and molecular target therapy in ovarian carcinomas. Hum Cell 18: $1-16$

van der Jagt MF, Sweep FC, Waas ET, Hendriks T, Ruers TJ, Merry AH, Wobbes T, Span PN (2006) Correlation of reversion-inducing cysteinerich protein with kazal motifs (RECK) and extracellular matrix metalloproteinase inducer (EMMPRIN), with MMP-2, MMP-9, and survival in colorectal cancer. Cancer Lett 237: 289-297

Vigneswaran N, Beckers S, Waigel S, Mensah J, Wu J, Mo J, Fleisher KE, Bouquot J, Sacks PG, Zacharias W (2006) Increased EMMPRIN (CD 147) expression during oral carcinogenesis. Exp Mol Pathol 80: 147-159

Weidner N (1995) Current pathologic methods for measuring intratumoral microvessel density within breast carcinoma and other solid tumors. Breast Cancer Res Treat 36: 169-180

Yang JM, O'neill P, Jin W, Foty R, Medina DJ, Xu Z, Lomas M, Arndt GM, Tang Y, Nakada M, Yan L, Hait WN (2006) Emmprin (CD147) confers resistance of breast cancer cells to anoikis through inhibition of bim. J Biol Chem 281: 9719-9727

Yoshida S, Shibata M, Yamamoto S, Hagihara M, Asai N, Takahashi M, Mizutani S, Muramatsu T, Kadomatsu K (2000) Homo-oligomer formation by basigin, an immunoglobulin superfamily member, viaits $\mathrm{N}$-terminal immunoglobulin domain. Eur J Biochem 267: 4372-4380 
Zheng HC, Sun JM, Wei ZL, Yang XF, Zhang YC, Xin Y (2003) Expression of Fas ligand and caspase- 3 contributes to formation of immune escape in gastric cancer. World J Gastroenterol 9: 1415-1420

Zheng H, Takahashi H, Murai Y, Cui Z, Nomoto K, Miwa S, Tsuneyama K, Takano Y (2006a) Pathobiological characteristics of intestinal- and diffuse-type gastric carcinoma in Japan: an immunostaining study on the tissue microarray. J Clin Pathol (in press)
Zheng H, Tsuneyama K, Cheng C, Takahashi H, Cui Z, Murai Y, Takano Y (2006b) Expressions of KAI1 and tenascin, microvessel density were closely correlated with liver metastasis of gastrointestinal adenocarcinoma. $J$ Clin Pathol (in press)

Zhou S, Liu C, Wu SM, Wu RL (2005) Expressions of CD147 and matrix metalloproteinase- 2 in breast cancer and their correlations to prognosis. Ai Zheng 24: 874-879 